\title{
Strength analysis of clavicle fracture fixation devices and fixation techniques using finite element analysis with musculoskeletal force input
}

\author{
Cronskär Marie ${ }^{1}$
}

Received: 19 July 2013 / Accepted: 26 March 2015 / Published online: 8 April 2015

(C) International Federation for Medical and Biological Engineering 2015

\begin{abstract}
In the cases, when clavicle fractures are treated with a fixation plate, opinions are divided about the best position of the plate, type of plate and type of screw units. Results from biomechanical studies of clavicle fixation devices are contradictory, probably partly because of simplified and varying load cases used in different studies. The anatomy of the shoulder region is complex, which makes it difficult and expensive to perform realistic experimental tests; hence, reliable simulation is an important complement to experimental tests. In this study, a method for finite element simulations of stresses in the clavicle plate and bone is used, in which muscle and ligament force data are imported from a multibody musculoskeletal model. The stress distribution in two different commercial plates, superior and anterior plating position and fixation including using a lag screw in the fracture gap or not, was compared. Looking at the clavicle fixation from a mechanical point of view, the results indicate that it is a major benefit to use a lag screw to fixate the fracture. The anterior plating position resulted in lower stresses in the plate, and the anatomically shaped plate is more stress resistant and stable than a regular reconstruction plate.
\end{abstract}

Keywords Clavicle $\cdot$ Finite element analysis $\cdot$ Multibody simulation $\cdot$ Bone plates $\cdot$ Fixation techniques

Cronskär Marie

marie.cronskar@miun.se

1 Department of Quality, Mechanics and Mathematics, Mid Sweden University, Akademigatan 1, 83125 Östersund, Sweden

\section{Introduction}

Fractures to the clavicle accounts for about $4 \%$ of all fractures [1] and $44 \%$ of all injuries to the shoulder girdle [2], but there is very differing opinions about the optimal treatment of clavicle fractures and there are great variations in treatment methods between countries [1]. Opinions differ on how often clavicle fractures should be operated on or treated non-operatively and about what kind of fixation device and technique to use when operating [3-5]. The interaction of muscles and ligaments in the shoulder region is complex. Consequently, it is difficult to perform experimental studies with realistic physical loading of the clavicle fixation. There are also inconsistencies in the results of existing biomechanical studies. Simplified loading cases such as axial compression, torsion, cantilever bending or three-point bending have mainly been used in the previous biomechanical studies, and it is likely that the inconsistency of the results is mainly a consequence of the difference in loading modes as also identified in [6] based on the literature review. Superior plate placement has been found to better prevent bending failure (in cantilever and three-point bending) than anteroinferior placement [7-9]. At the same time, the anteroinferior placement provided better stability in compression and torsion, in one of the studies [9]. In the studies using four-point bending, the anteroinferior plate placement also provided a more stable construct than the superior plate placement [6, 10]. Different previous studies also show different result regarding plating with locked or non-locked screws, and the results are dependent on the loading case, the plate placement and the use of bicortical or unicortical screws [5, 7, 9, 11]. More knowledge about what type of loading the clavicle and the plate is exposed to is needed, and one way of refining the load case and getting a better understanding of the loading of the clavicle and the 
bone-plate interaction is simulation, which has proven to be a useful tool in other parts of the body [12-16]. Previous FE models of clavicles mainly evaluate the response of intact clavicles without implants, simulating injury prediction in traffic accidents [17-21].

In [4], Favre used finite element (FE) modeling to compare the superior and anteroinferior position of a clavicle reconstruction plate under axial compression and cantilever bending with a simplified plate-bone interaction where the plate is attached to the clavicle by gluing the respective nodes in the contact interface. No screw holes and screws are included in the model. In other FE studies, one on lumbar spine stresses and one on acetabular implant stability, it has been found useful to include muscle and ligament forces derived from multibody simulation in the analysis $[22,23]$. Taylor et al. [6] also obtained results closer to reported clinical studies, while using multibody simulation as basis for building their experimental test rig. In a previous study, the author used a similar musculoskeletal model to obtain a realistic load case for input in the FE simulation of a clavicle with bone plate [24].

While importing the loading from the multibody simulation into the FE solver, in this case Abaqus (Dassault Systemes, Vélizy-Villacoublay, France), the load case file has to be combined, using a converter tool included in the AnyBody suit (AnyBody Technology, Aalborg, Denmark), with a mesh file of the bone. It results in an input file containing a mesh representation of the bone geometry, in Abaqus so-called orphan mesh. Since new screw holes have to be made in the mesh when changing plate design, an orphan mesh model requires a lot of work including: creating a new mesh, a new multibody simulation and rebuilding of the model each time a new plate is to be analyzed. This makes it very time-consuming to compare different fixation devices in this type of model. In the current study, the previous FE model [24] was modified to be a solid model of the bone to be more adapted for comparative fixation device studies.

The morphology of the clavicle bone varies greatly in shape and curvature [25] and even though there are many types of anatomically shaped plates on the market, the surgeon usually has to use precious surgery time to reshape the plate for a satisfying fit. The fit of the plate is important since it is positioned in an exposed area right under the skin, which can cause inconvenience to the patient. The FE model in this study can, after further development, be an important tool in future studies in the work with optimization of the plate shape of customized plates (based on bone CT data) and for analysis of the customized plate's strength compared to the current commercial plates.

The aims of this study were to:
1. Modify the previous model to make it more useful for comparison of different plates.

2. Perform sensitivity analysis to study the impact on resulting stresses and displacements due to simplifications of the model.

3. Use the modified model to perform comparative stress analyses between different clavicle fixation devices and strategies.

(a) LCP reconstruction plate with the pre-contoured LCP Sup-Ant clavicle plate.

(b) Anteroinferior versus superior plating position.

(c) Plating with and without a lag screw fixating the fracture gap.

\section{Methods}

\subsection{Background}

This study is a continuation of the work in [24], in which the method of combining the clavicle bone geometry with the muscle and ligament force inputs are explained in detail. In short, the bone model was created from a computed tomography scan (CT) of a left clavicle. A STL mesh representing the bone contour was created based on the grayscale values of the CT images using Mimics 14.11 software (Materialise, Leuven, Belgium). An oblique fracture with a $38^{\circ}$ angle was then simulated on the mesh, for more details, refer to [24]. Finally, the mesh was slightly modified to fit in the musculoskeletal simulation model. Later, the AnyBody Modeling System (AnyBody Technology, Aalborg, Denmark) was used to perform a multibody musculoskeletal analysis based on inverse dynamics on the clavicle model. The musculoskeletal simulation method is thoroughly described in Damsgaard et al. [26]. The output from the musculoskeletal analysis is an Extensible Markup Language (xml) file which was later combined with the STL mesh to form an input file containing bone geometry with muscle and ligament forces. The input file was imported into the Abaqus FE solver as a socalled orphan mesh model, which means that the geometry is defined by a non-changeable mesh instead of a changeable CAD-model which is later meshed inside the FE solver.

\subsection{Modeling of clavicle bone}

In order to easily compare different plates without having to perform new meshes and new multibody simulations for each scenario, a solid model of the bone mesh was created using RhinoResurf (Rhinoceros 4.0, McNeal 


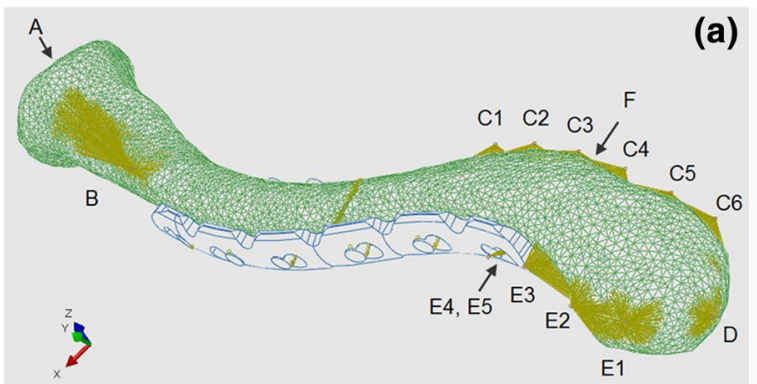

Fig. 1 a Orphan mesh model with LCP Recon plate in anteroinferior position and muscle and ligament attachments. For labels, $A-F$ see Fig. 2. The labels $A, E$ and $F$ are attachments not visible in current

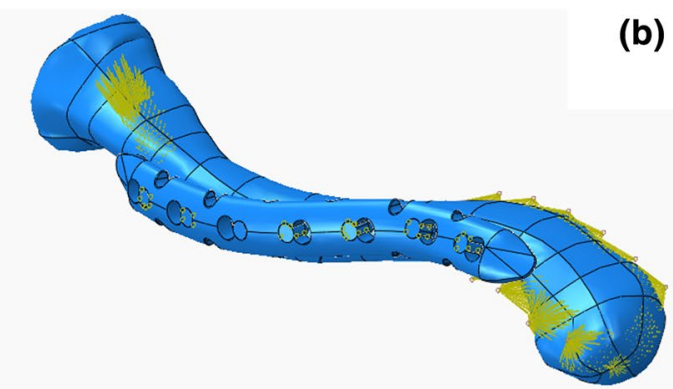

view [24]. b The corresponding solid model of the bone with a precontoured LCP Sup-Ant plate
Fig. 2 Arm position in the multibody musculoskeletal simulations and resulting forces from the simulations in [24]

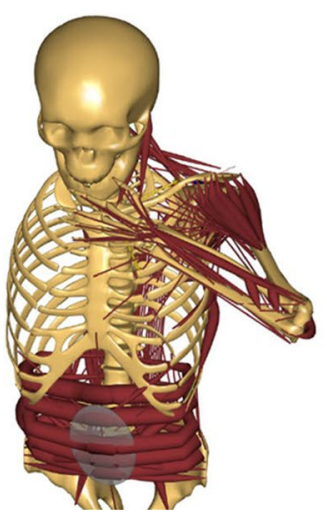

\begin{tabular}{|c|c|c|c|}
\hline \multicolumn{4}{|c|}{$\begin{array}{l}\text { Muscle and ligament forces on the clavicle from the multibody } \\
\text { musculoskeletal simulation }\end{array}$} \\
\hline \multirow[b]{2}{*}{ Muscle/ligament } & \multicolumn{3}{|c|}{ Muscle force components (N) } \\
\hline & $\mathrm{Fx}$ & Fy & $\mathrm{Fz}$ \\
\hline Lig. Conoid (F) & $-86,8$ & $-84,8$ & $-11,5$ \\
\hline Lig. Trapezoid & 0 & 0 & 0 \\
\hline M. Deltoid (E) & 59,2 & $-46,8$ & -25.8 \\
\hline M. Pect. major & 0 & 0 & 0 \\
\hline M. Sternocleidomastoid (B) & $-4,2$ & 14,2 & -1.5 \\
\hline M. Trapezius (C) & -2.8 & 22,4 & 30,5 \\
\hline Sternum contact force (A) & 11,1 & 13,4 & $-10,1$ \\
\hline Acromion contact force (D) & 23,4 & 86,5 & 18,3 \\
\hline
\end{tabular}

North America, USA), imported into the present FE model and oriented in the same position as the orphan mesh. A simulated fracture was created on the solid bone model in the same position as the fracture of the orphan mesh. The file with the solid bone, muscle attachment points and ligament attachment points (including direction and amplitude of the different reaction forces) was used as a starting model for the different analyses. New plates and screws were imported into that model. Then new screw holes were made in the solid bone, which was then meshed inside the FE solver. Finally, the muscle attachment points were coupled to muscle attachment surfaces created on the new mesh (Fig. 1).

In order to make the bone model as realistic as possible in the previous study, a representation of the trabecular bone was created based on the grayscale values in the CT images and a corresponding hole was created in the clavicle bone. It was later proven that the inner/trabecular bone had small impact on the bending resistance and the maximum stress in the plate was 483 and $481 \mathrm{MPa}$, respectively, in a model with a trabecular hole in the bone and one with cortical bone properties through all [24]. Therefore, this model is simplified to a solid bone model with cortical bone properties.

\subsection{Input forces to the FE simulations}

The musculoskeletal simulations were based on an early rehabilitation motion representing the first period of the healing process, when there is little or no healing in the fracture. After surgery, the patient is supposed to restrict the arm movements as much as possible. The position used in the simulations is the static position when holding a weight of $0.5 \mathrm{~kg}$, in front of the mouth (Fig. 2) representing a plausible loading case of a patient eating or drinking. In the FE model input file, the muscle forces derived from the musculoskeletal simulations were derived as concentrated forces at points representing the muscle and ligament attachments. Some muscles are divided into several parts, and each muscle force part is described by $x, y$ and $z$ force components. The point loads are projected on surfaces of the bone with an Abaqus called coupling constraints, coupling the point to the surface in all six degrees of freedom (dof). The surfaces represent the muscle and ligament attachment areas and are manually created in the FE model. In the previous study, the definition of these surfaces was based on clavicle anatomy in the literature $[27,28]$. Pictures of each surface were then saved and used as guidelines in following models to make sure that the deviations between the different models 
would be as small as possible. To confirm that small deviations in the surface representation would not have considerable impact on the calculations, a sensitivity analysis was performed where the only variable between the models were changes in the muscle and ligament attachment areas. Twelve analyses were performed in which the different muscle surface representations were varied according to size, position and with and without surfaces overlapping each other. The arm position and the input forces to the FE simulations coming from [24] are shown in Fig. 2. For corresponding muscle and ligament attachments, see Fig. 1.

\subsection{Investigated fixation devices and fixation techniques}

The plates analyzed in this study are the LCP Recon Plate 3.5 straight, 6 holes (Synthes, West Chester, USA) and the LCP Sup-Ant Clavicle Plate 3.5, 7 holes (Synthes). The plates were scanned, and 3D reconstructions were created. The LCP Recon plate was scanned and modeled as a straight reconstruction plate which was later bent in the SOLIDWORKS software (Dassault Systemes, Waltham, USA) to follow the bone contour, corresponding to the contouring often made by the surgeon during operation. The fit of the pre-contoured LCP Sup-Ant plate was considered to be sufficiently good, based on the subjective opinion of an experienced orthopedic surgeon, and was not modified after scanning. In all models, the screw holes were simplified and modeled without threads and countersinks. The material used for the plates and screws is a stainless steel (316 L) material, $\mathrm{E}=186.4 \mathrm{GPa}$ and Poisson's ratio 0.3 [29]. The 3D models of the plates were later imported into the FE-starting model in the universal Initial Graphics Exchange Specification (IGES) file format. The screws were modeled without threads and countersinks using the SOLIDWORKS software. In order to investigate different plating strategies, the LCP Recon plate was bent in two different ways to fit an anterior and a superior plating position. Finally, the three FE models including the different plates were also modeled with lag screws fixating the fracture.

The superior-positioned LCP Recon plate was the only plate in a position where it was possible to use one of the screw holes in the plate for fixation of a lag screw through the fracture. The LCP Sup-Ant and LCP Recon in anterior position were therefore only modeled using separate lag screws. For the same reason, one hole was left empty in the middle of the plate while modeling the superior-positioned LCP Recon plate without lag screw and with a separate lag screw. The different analyses are presented in Table 1.

\subsection{FE model details}

The muscle and ligament forces were, as mentioned previously, modeled as concentrated forces at points. Large
Table 1 Analyses in the study

\begin{tabular}{|c|c|c|c|}
\hline Analysis & Model & Position & $\begin{array}{l}\text { With or without lag } \\
\text { screw }\end{array}$ \\
\hline A1 & LCP Recon & Anterior & Without \\
\hline $\mathrm{A} 2$ & LCP Recon & Anterior & With \\
\hline A3 & LCP Recon & Superior & Without \\
\hline A4 & LCP Recon & Superior & With \\
\hline A5 & LCP Recon & Superior & $\begin{array}{l}\text { With lag screw through } \\
\text { plate }\end{array}$ \\
\hline A6 & $\begin{array}{l}\text { LCP Sup-Ant (pre- } \\
\text { contoured) }\end{array}$ & Sup-Ant & Without \\
\hline A7 & $\begin{array}{l}\text { LCP Sup-Ant (pre- } \\
\text { contoured) }\end{array}$ & Sup-Ant & With \\
\hline
\end{tabular}

muscles were divided into several parts. The points were coupled in all 6 dof to surfaces representing the muscle or ligament attachment areas on the bone. The plates, plate configurations and screws were imported as IGES files into the starting model, see chapter 2.2, oriented in the correct positions and used in Boolean operations to create appropriate screw holes in the solid bone model. After that, the solid bone model was meshed with a general element size of around $1.5 \mathrm{~mm}$ and with around 61,300 elements (91,800 nodes). The LCP Recon plate and LCP Sup-Ant plate were meshed with 39,692 and 45,944 elements, and 63,607 and 73,544 nodes, respectively. The bone, plates and screws were all meshed with quadratic (10 nodes) tetrahedral elements of type C3D10 (Abaqus). The number of elements in each screw was approximately 3000 (5000 nodes).

The surface of the plate screws was divided into two sections. The upper sections were assigned tie constraints (Abaqus) to the inner surfaces of the plate holes, simulating locked screws. The lower sections of the plate screws were coupled to the surfaces of the screw holes made in the bone model. In the models including lag screws (Table 1; A2, A4, A5 A7), a new screw hole was made in the bone model and tie constraints were assigned between the upper and lower parts of the screws and the holes surfaces in the two different bone fractions. The fracture is modeled like a fresh fracture without any healing between the two fractures edges. Both the tie constraint and the coupling constraints are rigid constraints.

The muscle and ligament forces (Fig. 2) almost create equilibrium in the model but to counteract rigid body motion, boundary conditions was assigned to small surfaces on the medial (sternal) and distal (acromial) ends of the clavicle bone. The medial end was locked in all linear translations and the rotation around the long axis of the clavicle bone. The distal end was locked in the transverse directions of the linear translations. The reaction 
Significant max stress (Von Mises, normalized)

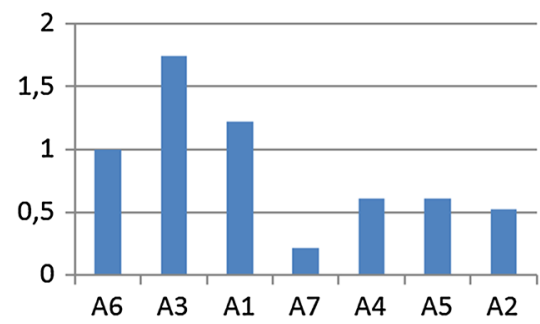

$(\mathrm{SA}) \quad(\mathrm{S}) \quad(\mathrm{A}) \quad(\mathrm{SA}, \quad(\mathrm{S}, \mathrm{L}) \quad(\mathrm{S}, \quad(\mathrm{A}, \mathrm{L})$

L)
Max displacement (magnitude, $\mathbf{m m}$ )

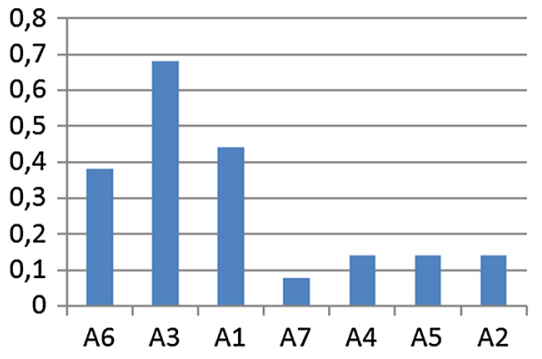

$(\mathrm{SA}) \quad(\mathrm{S}) \quad(\mathrm{A}) \quad(\mathrm{SA}, \quad(\mathrm{S}, \mathrm{L}) \quad(\mathrm{S}, \quad(\mathrm{A}, \mathrm{L})$

L)

LP)
Max Strain (Principal)

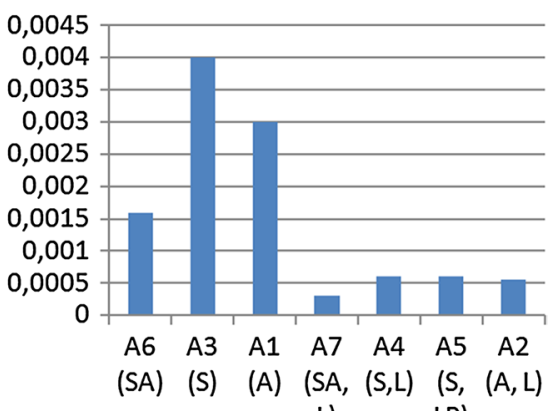

L)

LP)

Fig. 3 Maximum stress, displacement and strain in plate. S, superior (LCP Recon); A, anterior (LCP Recon); SA, superior-anterior (LPC SupAnt); L, lag screw; LP, lag screw through plate

forces in the boundary conditions were around $2.5 \mathrm{~N}$ in all seven models, demonstrating that the models are almost in equilibrium and the boundary conditions do not affect the results significantly. The previous orphan mesh model with a LCP Recon plate in anterior position and solid model with LCP Sup-Ant plate, both without lag screw, are shown in Fig. 1.

\subsection{Cortical bone material property}

In the current model, it was not applicable to use material data based on the CT density of the bone as in [30], due to reorientation and small rescaling of the bone mesh to suit the AnyBody model used in the musculoskeletal simulation [24]. Different material properties are used to define cortical bone in earlier FE studies [12, 15, 22, 23 ], and there is no easy way to assure what the correct material property is in each specific case. It was also assumed that the material property definition is not the most crucial input in this study since the purpose of the bone is to transfer forces from the muscles and ligaments to the plate, and it is primarily the stresses in the plate we are interested in. A sensitivity analysis was carried out to see how much the displacement in the bone and plate varies depending on the bone material used. Five different definitions of the Young's modulus $(E)$ were used in one model. One of the materials was orthotropic with $E=18 \mathrm{GPa}$ in longitudinal direction and $8 \mathrm{GPa}$ in transversal direction of the bone [31], and the other four were isotropic materials, $E=13.7 \mathrm{GPa}$ [15], $E=9 \mathrm{GPa}$ [32], $E=11 \mathrm{GPa}$ [33] and $E=15.7 \mathrm{GPa}$ [34]. The material used for the cortical bone in the rest of the analyses is the orthotropic material with a Young's modulus of $E=18 \mathrm{GPa}$ in the longitudinal direction of the bone and $E=8 \mathrm{GPa}$ in the transversal direction with a Poisson's ratio of 0.3 [31].

\section{Results}

\subsection{Comparison of fixation devices and techniques}

A comparison between the stress concentrations in the different plates and plating methods is shown in Fig. 3 . The maximum displacements and strains are summarized in the same figure. The resulting von Mises stress distributions in the plates are shown in Fig. 4 and summarized in Table 2. The most significant difference in stress distribution is between the analyses with a lag screw through the fracture gap (A2, A4, A5, A7) and the analyses without such a screw (A1, A3, A6), see Fig. 3. The use of lag screw resulted in much lower stresses in the plate and in stresses spread more evenly on the plate. Furthermore, it caused the screws to take more of the load but the maximum stress in the screws was still lower than the maximum stress in the plates without the use of lag screws. The differences in plate stress while using one of the plate screws to compress the fracture (A5) compared to using a separate lag screw (A4) were negligible. The anteroinferior plate position resulted in lower plate stresses (A1, A2) compared to the superior position (A3-A5), with a significant difference in the case without the use of a lag screw (A3 compared to A1) and a small difference in the case including the use of a lag screw (A4 and A5 compared to A2). Furthermore, the maximum stress in the precontoured LCP Sup-Ant plate (A6-A7) was lower than the LCP Recon plate (A1-A5) both when the Recon plate was placed in the superior and anteroinferior position and with and without the use of a lag screw through the fracture gap. This was expected since the pre-contoured plate is thicker and has no notches close to the fracture gap.

The maximum stresses in the bone while using the different plates and plating strategies are presented in Table 2 and Fig. 5. The maximum stress in the bone was higher in 


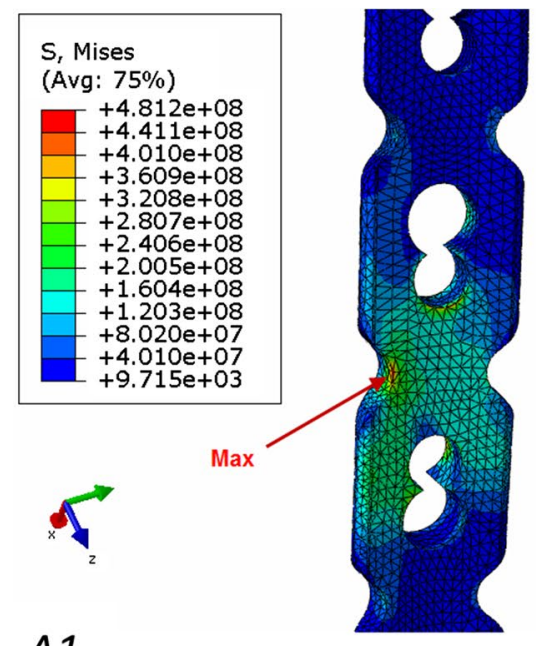

A1

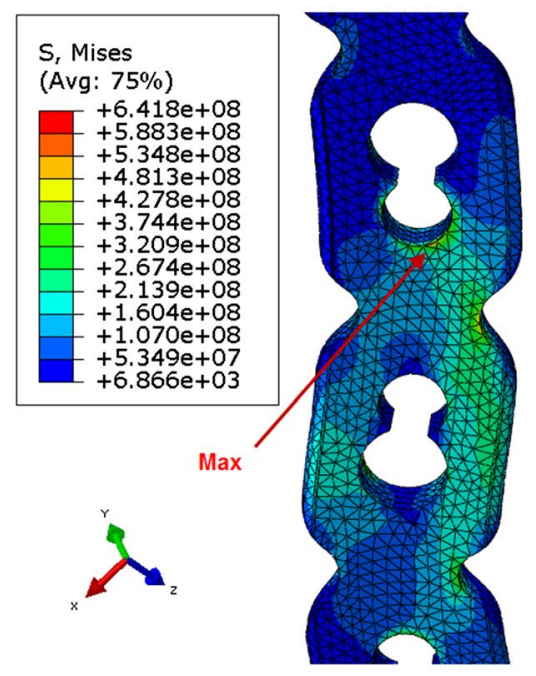

A3
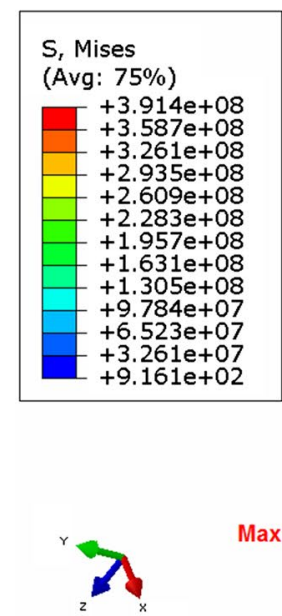

A6
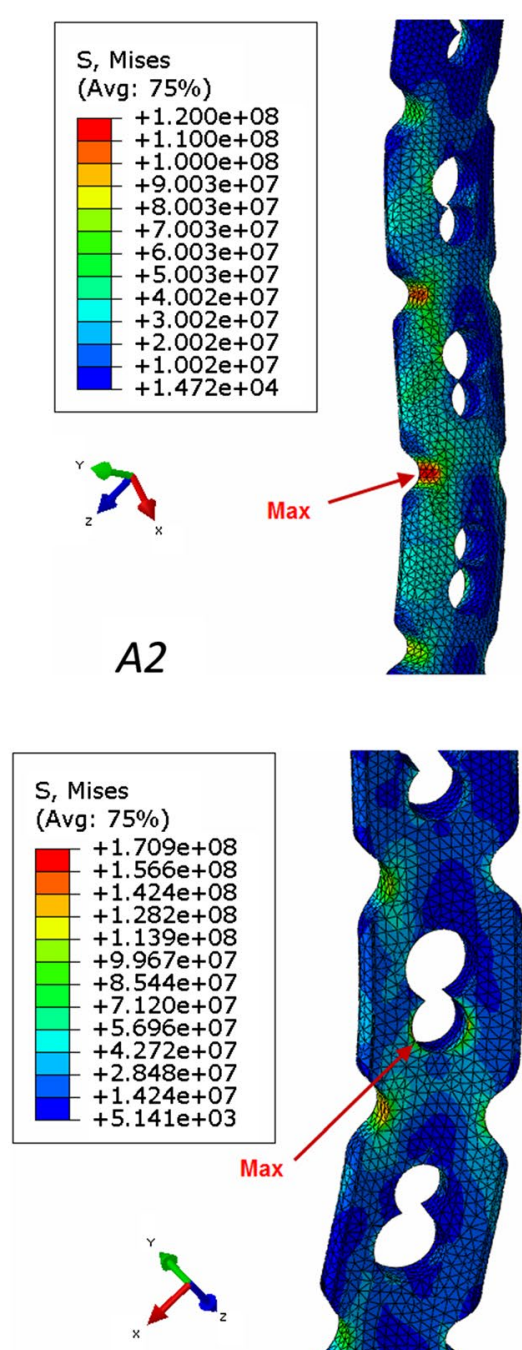

A4

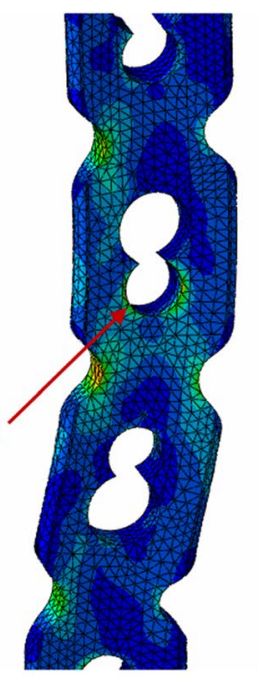

A5
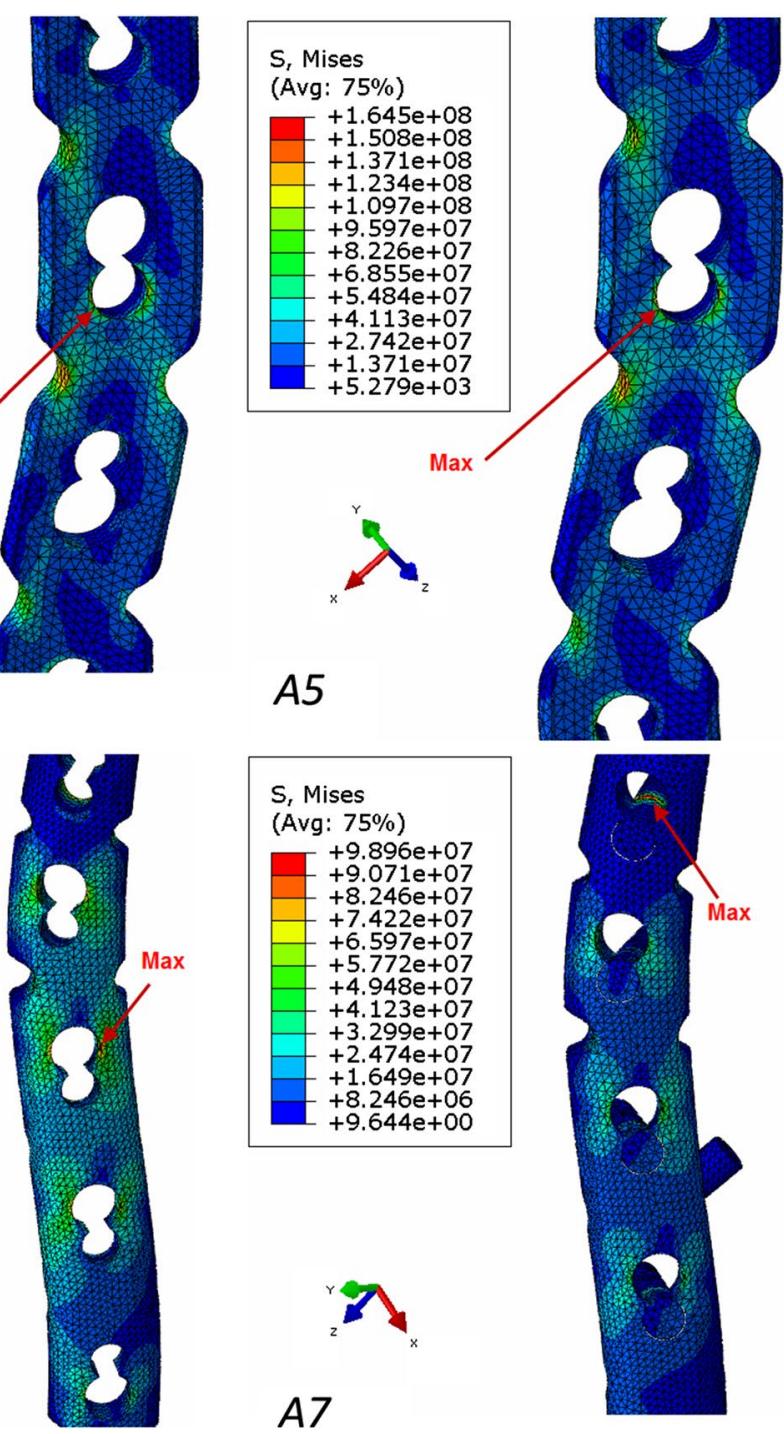

A7
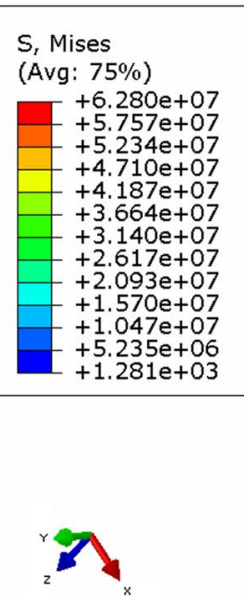

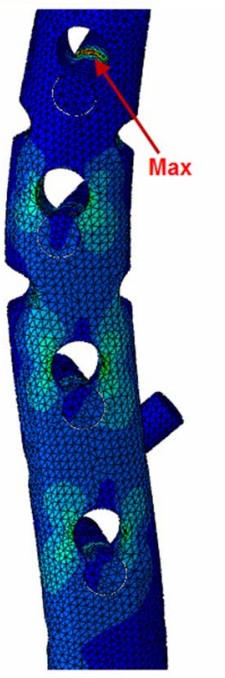

Fig. 4 Von Mises stresses in the seven analyses. See Tables 1 or 2 for explanation of the definitions A1-A7 
Table 2 Von Mises stress concentrations in relevant areas, excluding sharp holes edges

\begin{tabular}{|c|c|c|c|c|c|c|}
\hline \multirow[b]{2}{*}{ Without lag screw } & \multicolumn{2}{|l|}{ LCP Recon anterior } & \multicolumn{2}{|c|}{ LCP Recon superior } & \multicolumn{2}{|c|}{ LCP Sup-Ant (pre-contoured) } \\
\hline & $\begin{array}{l}\text { 160-280 } \\
\text { Max: } 481 \\
\text { Max in bone: } 13.8\end{array}$ & A1 & $\begin{array}{l}215-370 \\
\text { Max: } 642 \\
\text { Max in bone: } 26\end{array}$ & A3 & $\begin{array}{l}\text { 130-230 } \\
\text { Max: } 391 \\
\text { Max in bone: } 20\end{array}$ & A6 \\
\hline With lag screw & $\begin{array}{l}50-120 \\
\text { Max: } 120 \\
\text { Max in bone: } 41.2\end{array}$ & $\mathrm{~A} 2$ & $\begin{array}{l}70-140 \\
\text { Max: } 171 \\
\text { Max in bone: } 58\end{array}$ & A4 & $\begin{array}{l}20-50 \\
\text { Max: } 63 \text { ( } 98 \text { in screw) } \\
\text { Max in bone: } 25\end{array}$ & A7 \\
\hline With lag screw through plate & N/A & & $\begin{array}{l}60-140 \\
\text { Max: } 164 \\
\text { Max in bone: } 60\end{array}$ & A5 & N/A & \\
\hline
\end{tabular}

Line two: maximum stress in plate. Line three: maximum stress in bone (MPa)
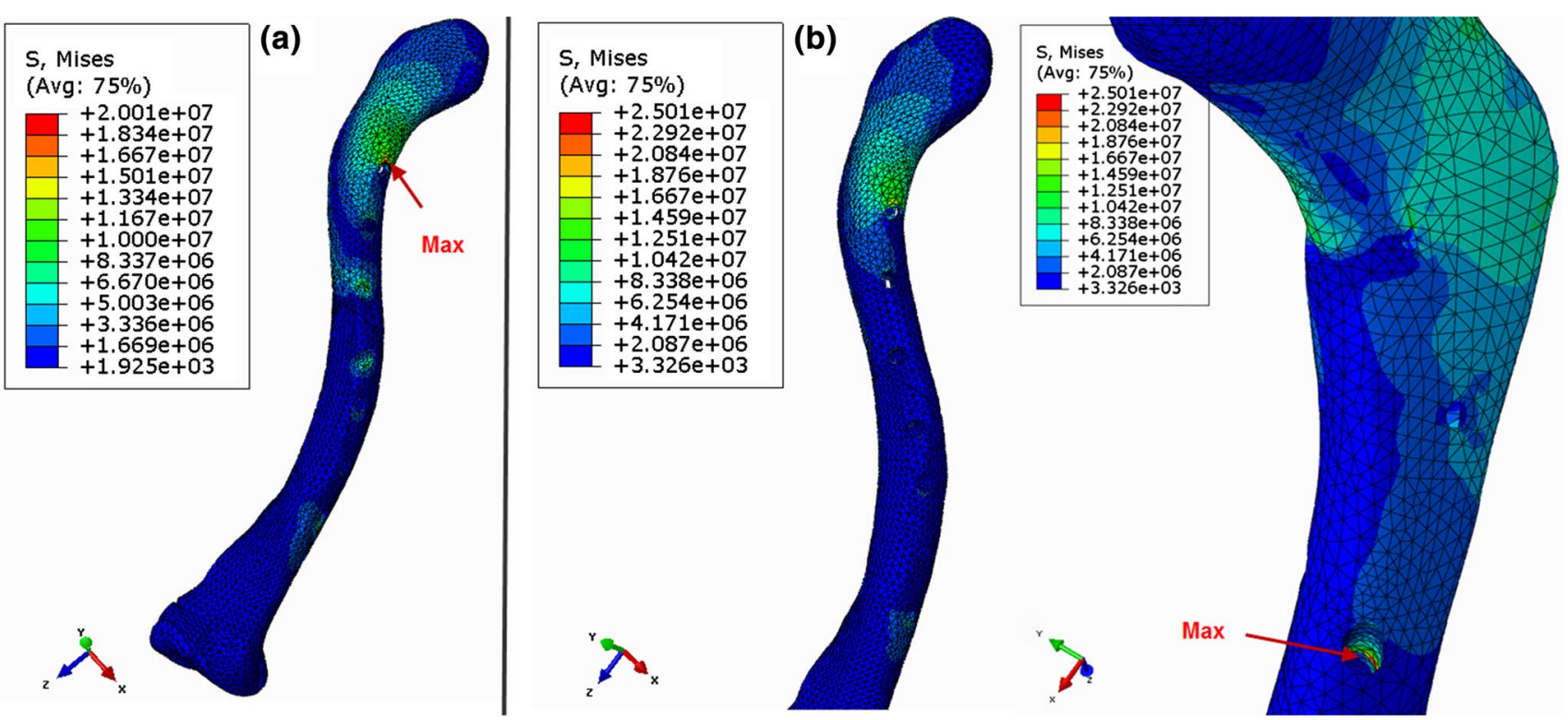

Fig. 5 Von Mises stresses in the bone when using a LCP Sup-Ant plate (plate hidden in picture). a The analysis A6 and b the analysis A7

the models with lag screw, but these are very local edge stresses. The models without lag screw had the maximum stress on the bones surface at the holes edge on the most distal hole and the ones with lag screw had the maximum inside the bone on one of the hole edges of the lag screw hole, see Fig. 5.

\subsection{Sensitivity analysis}

The stress in one of the middle notches of the anteroinferior-positioned LCP Recon plate was $460 \mathrm{MPa}$ in the original orphan mesh model [24]. Corresponding stress in the initial solid bone model was $440 \mathrm{MPa}$ [35] and later, with some small adjustments to the interaction definitions in the model, the corresponding stress was $460 \mathrm{MPa}$ in the same notch. The stress pattern was the same in all three analyses.
This shows that the model works in approximately the same way after the replacement of the orphan mesh.

The results from the sensitivity analyses of using different material properties for the cortical bone are shown in Fig. 6. The displacements in the bone and the plate both vary within $0.04 \mathrm{~mm}$ using the different bone materials.

The maximum resulting stresses and displacements depending on variations of the muscle attachment surface definitions are shown in Fig. 7. The stresses and displacements were almost exactly the same in all analyses with the exception of number eleven (Fig. 7), in which the muscle attachment surfaces had great overlaps which led to failure of the input forces and incorrect results. Also, in number eight (Fig. 7), the stresses and displacements are slightly higher due to overlapping surface definitions. 


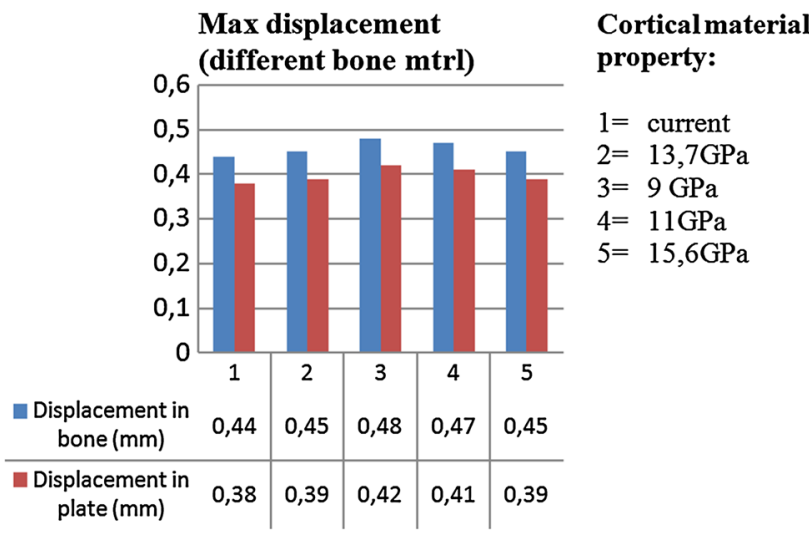

Fig. 6 Maximum displacements in bone and plate depending on the bone material property

\section{Discussion}

Opinions are divided regarding the optimal treatment of clavicle fractures but displaced mid-shaft fractures of the clavicle gain more and more importance in the field of orthopedic surgery [3]. When the clavicle fractures are treated operatively, there is also varying opinions about the best fixation device and strategy. A fixation plate placed on a clavicle is in a subcutaneous area, (especially when placed in a superior position), which makes the contouring and fit of the plate important to avoid hardware-induced discomfort [36]. It is quite common with remaining symptoms (sequelae), both after conservative treatment and operative treatment of clavicle fractures [37-39]. Better understanding of the loads on the clavicle and fixation devices is necessary to be able to develop fixation devises and techniques optimized to the preconditions and needs within clavicle fracture fixation. The most relevant types of investigations are clinical studies, but the drawbacks of these are that it is difficult to isolate parameters while comparing other parameters and it is difficult to carry out experimentation. Fabricating realistic experimental test rigs for cadaver studies and other biomechanical studies is time-consuming and also has some limitations in the possibility to isolate important parameters. FE simulation techniques are constantly developing and are able to quickly alter parameters in a controlled manner to provide mechanical responses. It can also be used in a more experimental way without any risks and with small increases in time and cost. This makes simulations a good complement to other types of investigations. However, one must keep in mind that the definition of; the load case, the material, and the interaction between the different parts of the assembly in FE simulations are simplifications of varying degrees and that the simulation results should be locally and globally validated toward appropriate biomechanical data obtained from experimental studies.

In summary, the main limitations in the study are as follows: first, the results of this study are based on this specific case, in the arm position presented, and should be considered as indicative results. In future studies, the simulations should be complemented with simulations in other arm positions. Second, the model is not validated toward experimental biomechanical studies. In this study, the model is used for relative analyses between different plates and plating strategies, and hence, the results are presented as the differences between the different strategies and not as absolute values. In the future, before using it to dimension, optimize or confirm the strength of a new plate designs, the model has to be validated toward experimental data. Further, the tie-constraints between bone and screws are simplified as completely rigid and the screws are modeled without threads and countersinks. Finally, there is no trabecular cavity in the bone allowing for bicortical screw
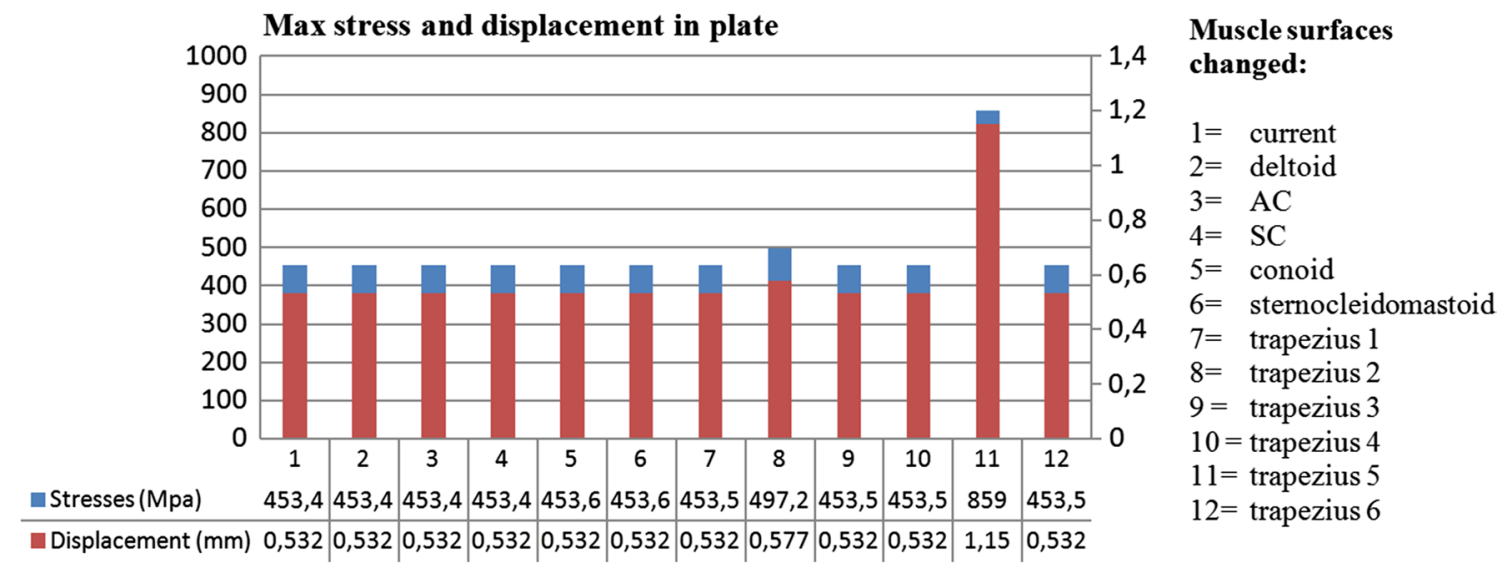

Fig. 7 Maximum stress and displacement in plate due to the definition of muscle and ligament attachment surfaces 
fixation but that simplification proved to have small impact on the resulting stresses in the plates.

Regarding the experimental biomechanical studies of anteroinferior contrasuperior plate positioning, previous results are contradictory but several studies show superior positioning to be advantageous, especially in bending tests. Celestre et al. [7] found that for the bending failure testing, the superior plate location had higher load to failure and bending failure stiffness. Harnroongroj and Vanadurongwans [40] reported that superior plating proved to provide more stability against bending moment than anterior plating when applied on clavicle bones without cortical defect, whereas on bones with cortical defect, the anterior plate position was found to provide more stability. Robertson et al. [9] found that in compression and torsion, anteroinferior plates were stiffer to superior (except for the nonlocked) and in cantilever bending, the superior plates had a significantly higher bending failure load and stiffness. Taylor et al. [6] focused on using a more realistic load case in their biomechanical studies, and their results indicate that inferior-medial (same as anteroinferior) plating provides improved construct stiffness.

Based on clinical experiences, several studies advocate anteroinferior plating [41-43] and one of them also emphasizes the use of lag screws [42]. Advantages of anteroinferior positioning are stable fixation, protection of infraclavicular structures and minimal incidence of implant prominence problems. Our results also indicate that it is favorable to use a lag screw to compress the fracture and to place the plate in an anteroinferior position. Though it is not always possible to attach a lag screw to fixate the fracture, for example, in the case of a comminuted fracture, it can be complicated. Furthermore, the pre-contoured plate without notches in the middle of the plate resulted in lower maximum stresses than the reconstruction plate in which the notches acted as stress raisers. The author has only found one previous FE study including clavicle bone and plate in the literature (loaded under axial compression and cantilever bending), and the results of this study were that anteroinferior positioning is advantageous and that special attention should be paid to achieve good fixation around the fracture and to avoid plate notches close to the fracture, which agrees well with results obtained in this study [4]. Present results also agree with the experimental studies using 4-point bending (which is the experimental loading most similar to the load case in the present simulation), which anteroinferior plate placement is biomechanically advantageous over superior plate placement $[6,10]$.

Designing the plate without notches and holes above the fracture and to use a lag screw to fixate the fracture when possible, both seem to significantly reduce the stress impact on the plate. While using customized plates, based on the bone contour and actual fracture as in $[44,45]$, the plate can be designed for the use of a lag screw and without stress raisers close to the fracture. That way, the stresses in the plate would be significantly lower and could probably enable a leaner plate design with maintained strength. In further work, customized plates designed based on these guidelines will be compared to commercial plates regarding mechanical response. Another interesting continuation is to study the screw-bone interaction more in detail, as in [46] and to find a way to include the risk of screw loosening in the model. The purpose with plate osteosynthesis is to achieve proper healing of the fracture, and hence, the best plate is a plate that contributes to fracture healing with minimized negative side effects. With better knowledge of the stress pattern in the plate, the plate can be designed with custom stiffness in different areas of the plate, enhancing the interfragmentary motion needed for secondary bone healing. But, before using the model for such purposes, it first has to be validated toward experimental studies.

In conclusion, the use of lag screw in the fracture gap had the highest impact on lowering the plate stresses compared to the plate position (anteroinferior compared to superior) and plate design (LCP Recon compared to precontoured LCP Sup-Ant). Using a lag screw in the fracture gap is also the clinical recommendation, but it is not always possible due to the type of fracture and the plate design. If a lag screw in the fracture gap could be guaranteed, the plate could have a leaner design. The anteroinferior plating position resulted in lower plate stresses in relation to superior position. The anteroinferior position also has clinical benefits such as a reduced risk of damaging vital structures when drilling and it entails less plate prominence. As expected, the pre-contoured LCP Sup-Ant also resulted in lower plate stresses than the LCP Recon plate, probably due to a plate design without notches close to the fracture and a greater plate thickness. The LCP Sup-Ant plate starts medially in an anterior position (with the benefits of easier drilling and less plate prominence problems) and ends in a more superior position with the advantages of less need to detach muscles than with anterolateral placement.

Acknowledgments This work was funded by the Swedish Agency for Economic and Regional Growth (Tillväxtverket) and the European Regional Development Fund. The author would also like to thank tutor Mats Tinnsten and assistant tutors Lars-Erik Rännar and Mikael Bäckström for their contribution to the study.

\section{References}

1. Dines D, Lorich D, Helfet D (2008) Solutions for complex upper extremity trauma, 1st edn. Thieme Medical Publishers, New York

2. Robinson CM (1998) Fractures of the clavicle in the adult: epidemiology and classification. J Bone Joint Surg (British) 80-B:476-484 
3. Eden L, Doht S, Frey S, Ziegler D, Stoyhe J, Fehske K, Blunk T, Meffert R (2012) Biomechanical comparison of the locking compression superior anterior clavicle plate with seven and ten hole reconstruction plates in midshaft clavicle fracture stabilisation. Int Orthop 36:2537-2543

4. Favre P, Kloen P, Helfet DL, Werner CML (2011) Superior versus anteroinferior plating of the clavicle: a finite element study. J Orthop Trauma 25:661-665

5. Renfree T, Conrad B, Wright T (2010) Biomechanical comparison of contemporary clavicle fixation devices. J Hand Surg Am 35:639-644

6. Taylor PR, Day RE, Nicholls RL, Rasmussen J, Yates PJ, Stoffel KK (2011) The comminuted midshaft clavicle fracture: a biomechanical evaluation of plating methods. Clin Biomech 26:491-496

7. Celestre P, Robertson C, Mahar A, Oka R, Meunier M, Schwarts A (2008) Biomechanical evaluation of clavicle fracture plating techniques: does a locking plate provide improved stability? J Orthop Trauma 22:241-247

8. Iannotti MR, Crosby LA, Stafford P, Grayson G, Goulet R (2002) Effects of plate location and selection on the stability of midshaft clavicle osteotomies: a biomechanical study. J Shoulder Elbow Surg 11:457-462

9. Robertson C, Celestre P, Mahar A, Schwartz A (2009) Reconstruction plates for stabilization of mid-shaft clavicle fractures: differences between nonlocked and locked plates in two different positions. J Shoulder Elbow Surg 18:204-209

10. Partal G, Meyers KN, Sama N, Pagenkopf E, Lewis PB, Goldman A, Wright TM, Helfet DL (2010) Superior versus anteroinferior plating of the clavicle revisited: a mechanical study. $\mathrm{J}$ Orthop Trauma 24:420-425

11. Little K, Riches P, Fazzi U (2012) Biomechanical analysis of locked and non-locked plate fixation of the clavicle. Injury 43:921-925

12. Ataç MS, Erkmen E, Yücel E, Kurt A (2009) Comparison of biomechanical behaviour of maxilla following le fort i osteotomy with 2- versus 4-plate fixation using 3d-fea: part 2: impaction surgery. Int J Oral Maxillofac Surg 38:58-63

13. Lin C-L, Lin Y-H, Chen A-Y (2006) Buttressing angle of the double-plating fixation of a distal radius fracture: a finite element study. Med Biol Eng Comput 44:665-673

14. Peleg E, Mosheiff R, Liebergall M, Mattan Y (2006) A short plate compression screw with diagonal bolts-a biomechanical evaluation performed experimentally and by numerical computation. Clin Biomech 21:963-968

15. Pérez MA, Prados-Frutos JC, Bea JA, Doblaré M (2011) Stress transfer properties of different commercial dental implants: a finite element study. Comput Methods Biomech Biomed Eng 15:263-273

16. Raja Izaham RMA, Abdul Kadir MR, Abdul Rashid AH, Hossain MG, Kamarul T (2012) Finite element analysis of puddu and tomofix plate fixation for open wedge high tibial osteotomy. Injury 43:898-902

17. Arregui-Dalmases C, Pozo ED, Duprey S, Lopez-Valdes FJ, Lau A, Subit D, Kent R (2010) A parametric study of hard tissue injury prediction using finite elements: consideration of geometric complexity, subfailure material properties, ct-thresholding, and element characteristics. Traffic Injury Prev 11:286-293

18. Bolte JH, Hines MH (2000) Shoulder response characteristics and injury due to lateral glenohumeral joint impacts, SAE Technical Paper

19. Duprey S, Bruyere K, Verriest J-P (2008) Influence of geometrical personalization on the simulation of clavicle fractures. J Biomech 41:200-207

20. Duprey S, Bruyere K, Verriest J-P (2010) Clavicle fracture prediction: simulation of shoulder lateral impacts with geometrically personalized finite elements models. J Trauma Acute Care Surg 68:177-182

21. Untaroiu C, Duprey S, Kerrigan J, Li Z, Bose D, Crandall J (2008) Experimental and computational investigation of human clavicle response in anterior-posterior bending loading-biomed 2009. Biomed Sci Instrum 45:6-11

22. Kunze M, Schaller A, Steinke H, Scholz R, Voigt C (2012) Combined multi-body and finite element investigation of the effect of the seat height on acetabular implant stability during the activity of getting up. Comput Methods Programs Biomed 105:175-182

23. Wong C, Rasmussen J, Simonssen E, Hansen L, De Zee M, Dendorfer $S$ (2011) The influence of muscle forces on the stress distribution in the lumbar spine. Open Spine J 3:21-26

24. Cronskär M, Rasmussen J, Tinnsten M (2013) Combined finite element and multibody musculoskeletal investigation of a fractured clavicle with reconstruction plate. Comput Methods Biomech Biomed Eng 18(7):740-748

25. Ahmad M, Trewhella MJ, Bayliss NC (2006) Study to describe the morphology of a series of clavicles \& the dimensions of its intramedullary canal. J Bone Joint Surg (British) 88(SUPPIII): 401

26. Damsgaard M, Rasmussen J, Christensen ST, Surma E, De Zee M (2006) Analysis of musculoskeletal systems in the anybody modeling system. Simul Model Pract Theory 14:1100-1111

27. Netter FH (2011) Atlas of human anatomy. Saunders/Elsevier, Philadelphia

28. Nowak J (2002) Clavicular fractures, epidemiology, union, malunion, nonunion. Faculty of Medicine, Uppsala

29. Disegi J (2009) Implant materials. Wrought $18 \%$ chromium-14\% nickel-2.5\% molybdenum stainless steel, 3rd edn. Synthes (USA), West Chester

30. Li Z, Kindig MW, Kerrigan JR, Kent RW, Crandall JR (2012) Development and validation of a subject-specific finite element model of a human clavicle. Comput Methods Biomech Biomed Eng 16:1-11

31. Kim S-H, Chang S-H, Son D-S (2011) Finite element analysis of the effect of bending stiffness and contact condition of composite bone plates with simple rectangular cross-section on the biomechanical behaviour of fractured long bones. Compos B Eng 42:1731-1738

32. Astier V, Thollon L, Arnoux PJ, Mouret F, Brunet C (2008) Development of a finite element model of the shoulder: application during a side impact. Int J Crashworthiness 13:301-312

33. Zhao J, Narwani G (2005) Development of a human body finite element model for restraint system R\&D applications. In: Proceedings of the 19th international technical conference on the enhanced safety of vehicles (ESV), Washington, DC, 6-9

34. Reilly DT, Burstein AH (1974) The mechanical properties of cortical bone. J Bone Joint Surg 56:1001-1022

35. Cronskär M, Bäckström M (2013) Modeling of fractured clavicles and reconstruction plates using cad, finite element analysis and real musculoskeletal forces input. In: 10th international conference on modelling and measurement in medicine and biology, Budapest, Hungary, 235-243

36. Vanbeek C, Boselli K, Cadet E, Ahmad C, Levine W (2011) Precontoured plating of clavicle fractures: decreased hardwarerelated complications? Clin Orthop Relat Res 469:3337-3343

37. Bostman O, Manninen M, Pihljamaki H (1997) Complications of plate fixation in fresh displaced midclavicular fractures. J Trauma 45:778-783

38. Ledger M, Leeks N, Ackland T, Wang A (2005) Short malunions of the clavicle: an anatomic and functional study. J Shoulder Elbow Surg 14:349-354

39. Nowak J, Holgersson M, Larsson S (2005) Sequelae from clavicular fractures are common. Acta Orthop 76:496-502 
40. Harnroongroj T, Vanadurongwan V (1996) Biomechanical aspects of plating osteosynthesis of transverse clavicular fracture with and without inferior cortical defect. Clin Biomech 11:290-294

41. Chen C-E, Juhn R-J, Ko J-Y (2010) Anterior-inferior plating of middle-third fractures of the clavicle. Arch Orthop Trauma Surg 130:507-511

42. Collinge C, Devinney S, Herscovici D, Dipasquale T, Sanders R (2006) Anterior-inferior plate fixation of middle-third fractures and nonunions of the clavicle. J Orthop Trauma 20:680-686

43. Kloen P, Werner CL, Stufkens SS, Helfet D (2009) Anteroinferior plating of midshaft clavicle nonunions and fractures. Oper Orthop Traumatol 21:170-179
44. Cronskär M, Rännar L-E, Bäckström M (2012) Implementation of digital design and solid free-form fabrication for customization of implants in trauma orthopaedics. J Med Biol Eng 32:91-96

45. Takahashi H, Shuto B, Katayama M, Nagashima H, Okumura M (2012) Design and application of tailor-made plates for treating fractures in small animals. In: 2012 4th international conference on awareness science and technology (iCAST), pp 243-247

46. Wieding J, Souffrant R, Fritsche A, Mittelmeier W, Bader R (2012) Finite element analysis of osteosynthesis screw fixation in the bone stock: an appropriate method for automatic screw modelling. PLoS ONE 7:e33776 